\title{
Análise Faunística e Flutuação Populacional da Dipterofauna de Ecossistemas da Área de Proteção Ambiental do Araripe, Barbalha, CE
}

\author{
Francisco Roberto de Azevedo1 ${ }^{\bowtie}$, Raul Azevedo ${ }^{1}$, Cícero Antônio Mariano dos Santos², \\ Eridiane Silva Moura² \& Daniel Rodrigues Nere ${ }^{3}$
}

1. Universidade Federal do Cariri, e-mail: razevedo@ufc.br (Autor para correspondência ${ }^{\varpi}$ ), raulbiologo@gmail.com. 2. Universidade Federal de Viçosa, e-mail: anttony.ms@gmail.com, eridianedasilvamoura18@yahoo.com.br. 3. Secretaria de Desenvolvimento Agrário, e-mail: r.nere@agronomo.eng.br.

\section{EntomoBrasilis 8 (2): 117-124 (2015)}

Resumo. Os Diptera podem ser hematófagos, detritívoros, vetores de doenças, pragas importantes de plantas cultivadas, predadores ou parasitoides de insetos-praga. Algumas espécies polinizam plantas importantes e outras atacam ervas daninhas. Objetivando verificar a análise faunística e a flutuação populacional desses insetos nos ecossistemas da Área de Proteção Ambiental do Araripe, em Barbalha-CE, foram realizadas coletas semanais na estação seca e chuvosa, por meio da armadilha McPhail, iscada com suco de goiaba. Os Sarcophagidae e Drosophilidae são as famílias mais abundantes tanto na estação seca como na chuvosa, nos quatro ecossistemas da Área de Proteção Ambiental do Araripe. Os Sarcophagidae dominam e ocorrem frequente e constantemente em todos os ecossistemas da estação seca da Área de Proteção Ambiental do Araripe, enquanto que os Drosophilidae apesar de dominarem e ocorrerem constantemente, são mais frequentes na área agrícola e no Arajara Park devido à ação antrópica que altera esses ecossistemas para a implantação de culturas agrícolas e áreas de lazer. Os Drosophilidae dominam e ocorrem frequente e constantemente em todos os ecossistemas da estação chuvosa da Área de Proteção Ambiental do Araripe, enquanto que os Sarcophagidae, apesar de dominarem e ocorrerem constantemente, são mais frequentes na área agrícola e na mata úmida devido à ação antrópica obtida pelas irrigações das culturas agrícolas da área agrícola associada com maior umidade pelo microclima favorável encontrado na mata úmida. Os picos populacionais dos dípteros que predominam nos ecossistemas da Área de Proteção Ambiental do Araripe ocorrem nos meses chuvosos associados a baixas temperaturas.

Palavras-chave: Antropização; Coleta de insetos; Diversidade de insetos; Moscas.

\section{Faunal Analysis and Populational Fluctuation of Dipterofauna of Ecosystems from Área de Proteção Ambiental do Araripe}

Abstract. Diptera can be hematophagous, scavenger, and transmitter diseases, important pest to cultivated plants, predators or parasitoids of pestinsects. Some are pollinate important plants and others attack weeds. Aiming to evaluate the faunal analysis and populational fluctuation of these insect at ecosystems of "Área de Proteção Ambiental do Araripe" in Barbalha-CE, weekly surveys were been realized during dry and wet season using McPhail traps, using guava juice as a bait. Sarcophagidae and Drosophilidae were more abundant in dry and rainy season, with a major number of individuals collected during a rainy season in each ecosystem of the "Área de Proteção Ambiental do Araripe". The Sarcophagidae family dominates and occurs frequently and constantly in all ecosystems of dry season, while individuals of Drosophilidae, despite of be dominant and occurs constantly, are more frequent in agricultural area and Arajara Park due to anthropogenic action which changes these ecosystems for insert agricultural cultures and recreational areas. Drosophilidae dominates and occurs frequently and constantly in all ecosystems during rainy season, while Sarcophagidae in despite of dominate and occurs constantly, are more frequent at agricultural area and humid forest due anthropogenic action got by irrigations associated with a major humidity caused by favorable microclimate founded in rainforest. The Diptera populations picks predominate in all ecosystems of the "Área de Proteção Ambiental do Araripe" occurs at rainy months associated if low temperatures.

Keywords: Anthropization; Collection of insects; Flies; Insect diversity.

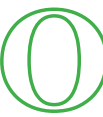

s Diptera são relativamente pequenos, de corpo mole, alguns são minúsculos, porém muitos têm grande importância econômica. Os pernilongos, borrachudos, mosquitos-pólvora, mutucas e moscas-de-estábulos sugam o sangue e constituem pragas sérias de humanos e animais. A maioria das moscas hematófagas e algumas moscas detritívoras, como as moscas domésticas e as varejeiras são vetores de doenças. Os organismos causadores de malária, febre tifoide, disenteria e outras doenças são transportados e distribuídos por espécies de Diptera. Algumas moscas, como as moscas das frutas são pragas importantes de culturas agrícolas. No entanto, existem moscas detritívoras úteis, assim como, predadores ou parasitoides de insetos-praga. Algumas polinizam plantas importantes e outras atacam ervas daninhas (TRIPLEHORN \& JOHNSON 2011).

De acordo com Silveira NeTo (1995), a análise faunística permite a avaliação do impacto ambiental, tendo por base espécies de insetos como indicadores ecológicos, observando-se que há interferência na fauna entomológica da comunidade analisada devido às mudanças e destruição da vegetação, fato este que vem sendo observado nos últimos anos na APA-Araripe devido à exploração imobiliária, turismo, mineração e outras ações antrópicas. Assim, os inventários de levantamento e identificação de insetos nos ecossistemas permitem as prevenções ou remediações de impactos nos diferentes ambientes. Isto caracteriza a importância destes estudos a fim de que se identifiquem certas famílias bioindicadoras potenciais. Pois assim, à medida que ocorre o resgate da diversidade e equilíbrio ambiental também os insetos respondem em diversidade e densidade, cumprindo a sua função indicadora (WINk et al. 2005).

Ferraz (2011) informa que estudos de ecologia de dípteros em ambientes florestais fragmentados apresentam-se ainda em pequeno número, portanto levantamentos da biodiversidade de áreas florestais são fundamentais, principalmente, para conhecer as espécies e/ou famílias que ocupam esses habitats, seus padrões 
T de distribuição (biogeografia), identificar aquelas que podem atuar como bioindicadoras ambientais e conhecer a dispersão e a adaptabilidade das exóticas, fornecendo informações fundamentais para a elaboração de políticas de conservação. Por isso, levantamentos faunísticos de Diptera na região Neotropical são essenciais, pois apenas uma pequena parte da diversidade biológica do grupo é conhecida, com estimativas de que o número de espécies de dípteros seja muitas vezes maior que a quantidade conhecida (TAuHYL \& GuIMARÃES 2012).

Alguns trabalhos têm mostrado que esses insetos podem indicar ação antrópica como a alteração da paisagem natural em uma área de Floresta Atlântica que gera mudanças que favorecem o desenvolvimento e proliferação de determinadas espécies de Culicidae (Anjos \& Navarro-Silva 2008). Efeitos de borda de fragmento florestal sobre populações de Calliphoridae (FurusAwA \& Cassino 2006; Ferraz et al. 2010). Padrões populacionais de Tephritidae em um fragmento de floresta semidecídua (PEREIRA 2008).

Considerando-se que os ecossistemas da Área de Proteção Ambiental do Araripe são peculiares, devido à sua diversidade e origem a partir de outras grandes regiões fitogeográficas, objetivou-se com essa pesquisa realizar uma análise faunística dos dípteros nesses ecossistemas e verificar a flutuação populacional das famílias mais abundantes, utilizando a armadilha tipo McPhail.

\section{MATERIAL E MÉTODOS}

Área de estudo. A pesquisa foi realizada na Área de Proteção Ambiental da Chapada do Araripe, em Barbalha-CE, de 03 de setembro de 2010 a 22 de fevereiro de 2011 (estação seca) e de 01 de março a 30 de agosto de 2011 (estação chuvosa).

Foram avaliados quatro ecossistemas, sendo uma Mata úmida, um Fragmento de mata, uma Área agrícola e uma Área de lazer (Arajara Park). A Mata úmida (07 13 '56"S e $39^{\circ} 22$ '12,7"W), pertencente ao bioma da Floresta Subperenifólia Tropical PluvioNebular, com 1.063.000 ha, com alto grau de conservação, constituído por vegetação lenhosa de médio porte, com alguns elementos com dossel alcançando até $15 \mathrm{~m}$, apresentando um sub-bosque composto por regeneração natural, muito densa. O Fragmento de mata ( $07^{\circ} 10^{\prime} 47^{\prime \prime}$ S e $39^{\circ} 23^{\prime}$ oo"W), também do bioma Floresta Subperenifólia Tropical Pluvio-Nebular, com 16,94 ha, apresenta-se bastante antropizado e continha áreas desmatadas para a criação de gado, havendo pastos de capim elefante (Pennisetum purpureum Schumacher), riachos com terrenos pedregosos, solo arenoso e plantas arbustivas. A Área agrícola (07 $19^{\circ} 50,5$ ”S e $39^{\circ} 24^{\prime} 40,9$ ” W), com 9,68 ha, continha laranjeiras (Citrus sinensis [L.] Osbeck), mangueiras (Mangifera indica L.), coqueirais (Cocos nucifera L.) e bananeiras (Musa spp.), além do cultivo do milho (Zea mays L.), feijão (Vigna unguiculata [L.] Walp.), mandioca (Manihot esculenta Crantz) e hortaliças como coentro (Coriandrum sativum L.), pimentão (Capsicum annuum L.) e cenoura (Daucus carota L.) na estação chuvosa, além da criação de animais domésticos como galinhas, patos e porcos. O Arajara Park ( $07^{\circ} 19^{\prime} 59,1^{\prime}$ 'S e $39^{\circ} 24$ ' 39,3 ”W) com 76 ha, continha mata úmida preservada e também locais com chalés, restaurante, tapiocarias, pesque-e-pague, parques aquáticos, trilhas, além de grutas e nascentes que percorriam todo o balneário.

Armadilhas e coletas. Foram instaladas quatro armadilhas em cada tipo de ecossistema, dispostas em duas fileiras de duas armadilhas, espaçadas de $50 \mathrm{~m}$ uma da outra $\left(2.500 \mathrm{~m}^{2}\right.$ de área amostrada), tendo como isca atrativa, $400 \mathrm{~mL}$ de suco de goiaba a $25 \%+10 \%$ de açúcar cristal e elas foram instaladas dentro da copa das árvores e/ou arbustos, em local sombreado e a uma altura média de 2,0 $\mathrm{m}$ do solo.

Em cada amostragem semanal $(\mathrm{N}=4)$, as armadilhas permaneceram operantes por sete dias e a coleta dos insetos foi feita semanalmente, com concomitante substituição da isca atrativa. Ao todo, realizaram-se quatro coletas mensais em cada ecossistema e em cada estação do ano, totalizando 24 coletas/ estação, sendo, portanto, feitas coletas durante o ano inteiro. Os insetos coletados foram identificados em nível de ordem e família, utilizando-se chaves ilustradas de identificação (Buzzi 2008; TRIPLEHORN \& JOHNSON 2011).

Análise faunística. Os ecossistemas foram comparados através dos seguintes índices faunísticos: frequência, dominância, abundância e constância (SILveira Neto et al. 1976) e os cálculos realizados por meio de planilhas do programa Excel.

\section{RESULTADOS E DISCUSSÃO}

\section{Índices faunísticos dos dípteros coletados na estação} seca. Nos quatro ecossistemas foi amostrado, na estação seca, um total de 14 famílias da ordem Diptera, sendo a família Sarcophagidae a mais numerosa, seguida de Drosophilidae (Tabela 1). Dos 62.421 indivíduos capturados nessa estação, $34,51 \%$ foram encontrados no fragmento, 31,45\% na área agrícola, 19,62\% na mata úmida e 14,42\% no Arajara Park. Foram encontrados 7.929 indivíduos de Otitidae, bem representados

Tabela 1. Número total de indivíduos das famílias de Diptera capturadas na estação seca nos quatro ecossistemas da Área de Proteção Ambiental do Araripe, Barbalha-CE, de 03 de setembro de 2010 a 22 de fevereiro de 2011.

\begin{tabular}{|c|c|c|c|c|c|}
\hline \multicolumn{6}{|c|}{ Ecossistemas } \\
\hline Famílias & $\begin{array}{c}\text { Fragmento de } \\
\text { mata } \\
\end{array}$ & Área agrícola & Mata úmida & Arajara Park & Total \\
\hline Bibionidae & 123 & 80 & 10 & 66 & 279 \\
\hline Bombyliidae & o & o & o & 4 & 4 \\
\hline Calliphoridae & 2.505 & 1.867 & 680 & 402 & $5 \cdot 454$ \\
\hline Chloropidae & 665 & 675 & 145 & 265 & 1.750 \\
\hline Dolychopodidae & 2 & o & o & 2 & 4 \\
\hline Drosophilidae & 4.919 & 6.652 & 1.562 & 3.362 & 16.495 \\
\hline Lonchaeidae & 114 & 75 & 1 & 22 & 212 \\
\hline Micropezidae & 95 & 33 & 22 & 64 & 214 \\
\hline Muscidae & 2.415 & 2.796 & 460 & 1.064 & 6.735 \\
\hline Otitidae & 2.910 & 933 & 3.539 & 547 & 7.929 \\
\hline Sarcophagidae & 6.560 & $5 \cdot 311$ & 4.214 & 2.640 & 18.725 \\
\hline Stratiomyiidae & o & o & 1 & 1 & 2 \\
\hline Tephritidae & 1.230 & 1.202 & 383 & 562 & $\mathbf{3 \cdot 3 7 7}$ \\
\hline Tipulidae & 2 & 5 & 1.232 & 2 & 1.241 \\
\hline Total & 21.540 & 19.629 & 12.249 & 9.003 & 62.421 \\
\hline
\end{tabular}


por Euxesta spp., devido à presença de gramíneas em todos os ecossistemas, principalmente na área agrícola em decorrência da presença da cultura do milho, já que é uma praga que ataca grãos em formação. De acordo com Souto et al, 2011, esse inseto tem distribuição tropical e subtropical no hemisfério ocidental e do mesmo modo tem sido considerada uma das principais pragas agrícolas da cultura do milho junto com Helicoperva zea (Boddie) e Spodoptera frugiperda (Smith). Os danos das larvas da mosca às gramíneas incluem o consumo dos estilo-estigmas, reduzindo a polinização; ausência de grãos na ponta da espiga devido ao alimento da larva; destruição dos grãos em desenvolvimento; aumento da vulnerabilidade da espiga a patógenos e redução da qualidade do grãos (GALLO et al. 2002).

Foram coletados 6.735 indivíduos de Muscidae e 5.454 de Calliphoridae. Os muscídeos são um grupo grande com 620 espécies e seus membros estão em quase todos os lugares e muitos são pragas importantes. A mosca doméstica desenvolvese em todos os tipos de sujeiras e frequentemente é muito abundante, transmitindo febre tifoide, disenteria, bouba, antraz e algumas formas de conjuntivite. A mosca da face é uma praga importante do gado, assim como, a mosca do estábulo e a do chifre (TRIPLEHORN \& JOHNSON 2011).

Já os califorídeos têm um importante papel na ciclagem de nutrientes no ambiente terrestre, uma vez que suas larvas atuam como decompositoras da matéria orgânica. Esta característica pode ser utilizada para estudos de entomologia forense (MourA et al. 1997) e acelerando processos de cicatrização na terapia larval (Neves 2005). Podem atuar também como polinizadores (Silva et al. 2001), veiculadores de patógenos (GrEENBERG 1973) e agentes causadores de miíases em vertebrados vivos (GUIMARÃES et al, 1983). Esses dípteros possuem grande capacidade de adaptação ao ambiente modificado pelo homem, sendo algumas espécies classificadas como sinantrópicas (FuRUSAWA \& CASSINO 2006). Como existiam animais domésticos e/ou silvestres em estado de decomposição nesses ecossistemas, isso explica a grande abundância dessas duas famílias. Algumas espécies de moscas hematófagas da família Muscidae, como Stomoxys calcitrans L., apresentam pouca especificidade quanto aos seus hospedeiros alimentando de diversas espécies de mamíferos e aves (BitTencourt \& Moya BorJa 2000) e como se trata de áreas propensas a existência de diversas espécies dos animais citados, justifica-se assim, o número de muscídeos coletados que foram atraídos pela substância açucarada do suco de goiaba e não pela sua preferência natural que é a hematofagia.

No entanto, apenas 3.377 indivíduos de Tephritidae foram constatados, quando comparado com as demais famílias. Os adultos dos tefritídeos são encontrados em flores ou na vegetação e são conhecidas como moscas-das-frutas, cujas larvas são fitófagas, alimentando-se de vários tipos de frutas (TRIPLEHORN \& JoHnson 2011). Provavelmente existiam nesses ecossistemas frutas hospedeiras cultivadas e/ou silvestres em processo de frutificação e/ou maturações que por sua vez fizeram com que as populações desses insetos se tornassem mais dispersas e não fossem tão atraídos pelo suco de goiaba.

Apesar das armadilhas McPhail terem sido desenvolvidas com o objetivo de capturar e monitorar moscas-das-frutas (Diptera: Tephritidae) (UCHÔA-FERNANDES et al. 2003) devido ao odor exalado pelos diversos tipos de atrativos utilizados (STEYsKaL 1977), diversos trabalhos têm demonstrado a eficiência de captura de outras famílias de Diptera, assim como outras ordens de insetos, tais como Coleoptera, Hymenoptera e Lepidoptera. $\mathrm{Na}$ região do Cariri cearense, AzEvEdo et al. (2011) coletaram diferentes ordens e famílias de insetos na Floresta Nacional do Araripe, dentre elas, Coleoptera, Hymenoptera e Lepidoptera, exemplificando assim a capacidade que essa armadilha de capturar uma grande diversidade de insetos. SANTos et al. (2012) coletaram 83.127 insetos utilizando essa mesma armadilha. Além dos dípteros, outras ordens foram capturadas como os
Coleoptera/Hymenoptera ( 7 famílias), Hemiptera (6 famílias), Orthoptera (4 famílias) e Neuroptera/Blattodea ( 1 família), no mesmo bioma estudado pelos autores citados anteriormente.

$\mathrm{Na}$ presente pesquisa observou a captura de alguns insetos de grande porte como mariposas e borboletas que morreram por não conseguir sair da mesma. Devido ao intervalo de tempo entre as coletas (sete dias), tais indivíduos entraram em estado de putrefação, contribuindo assim como atrativo para Calliphoridae. A abundância elevada desses insetos nas armadilhas se deve ao comportamento alimentar dos mesmos, visto que são os maiores consumidores de carcaças de animais e por permanecerem na carcaça em decomposição por longos períodos (BRAACK 1987; CATTS \& GoFF 1992).

No fragmento, os dípteros da família Sarcophagidae foram os mais frequentes (30\%), seguida de Drosophilidae (23\%) e Otitidae (14\%). Todas essas famílias foram dominantes e constantes, mas somente Otitidae foi muito abundante (Tabela 2). Dentro dessa família temos a morfoespécie Euxesta spp. que ataca um número variado de espécies de gramíneas e como nesse ecossistema existia uma grande plantação de capim elefante (Pennisetum purpureum Schum), isso explica a sua grande abundância por ser um ambiente modificado pelo homem para a criação de gado de leite. Por ser um ecossistema habitado também por alguns animais silvestres, já que é uma transição da mata úmida e apresenta frutos característicos da região, os sarcofagídeos e drosofilídeos têm sua função biológica na decomposição da matéria orgânica do fragmento, porque atuam sobre carcaças de animais silvestres e sobre frutos fermentados, respectivamente.

Os maiores picos populacionais dos sarcofagídeos e drosofilídeos ocorreram nos meses de janeiro e fevereiro em decorrência das maiores precipitações de 351,5 e 294,2 mm, respectivamente, associadas às baixas temperaturas de 25,95 e $25,4{ }^{\circ} \mathrm{C}$, respectivamente (Figura 1). DiAs et al, (1984) ressaltam que a umidade local do solo influencia a velocidade da decomposição da matéria orgânica tornando o ambiente mais propício para sarcofagídeos, desse modo, contribuindo assim para a dominância dos espécimes dessa família.

Como no Cariri cearense existe uma grande diversidade de frutos silvestres, como o pequi (Caryocar brasiliense Cambess.), a seriguela (Spondias purpurea L.), o cajá (Spondias lutea L.), o araçá (Psidium araca Raddi), a mangaba (Hancornia speciosa Gomes), o juazeiro (Ziziphus joazeiro Mart.), a carnaúba (Copernicia prunifera (Mill.) H.E. Moore), o maracujá (Passiflora edulis Sims) e outras, isso faz com que esses insetos tenham grande importância na ciclagem de nutrientes, nesse ecossistema da estação seca.

Os Calliphoridae e Muscidae encontrados com frequências de 12\% e $11 \%$, respectivamente, foram constantes no fragmento e apesar da primeira estar dispersa e a segunda ser de ocorrência comum, são insetos necrófagos e juntamente com os sarcofagídeos, são os primeiros a chegarem às carcaças dos animais, minutos após a morte, para a realização de oviposições e alimentação dos adultos (Sмiтh 1986), permanecendo na carcaça ao longo dos estádios de decomposição (CATTS \& GofF 1992). Como no fragmento existe uma grande diversidade de animais silvestres mortos é de suma importância a presença dessas famílias para a ciclagem de energia no ecossistema. Tal importância dos califorídeos e respectiva abundância podem ser exemplificadas no trabalho de LEANDRo \& D'Almeida (2005), onde esses insetos representaram mais de $65 \%$ dos espécimes encontrados. Famílias como Calliphoridae e Sarcophagidae vêm sendo utilizadas em estudos de cunho ecológicos, visando determinar os "Efeitos de Borda" em fragmentos florestais. Desse modo, por meio do Índice de Sinantropia utilizado por FurusaWA \& CASSINO (2006), foi possível associar a composição da fauna de Calliphoridae de um fragmento de mata secundária associado aos efeitos de borda. Mais recente, a ocorrência de determinados gêneros como 
Tabela 2. Análise faunística das famílias de Diptera capturadas na estação seca em quatro ecossistemas da Área de Proteção Ambiental do Araripe, Barbalha-CE, de 03 de setembro de 2010 a 22 de fevereiro de 2011.

\begin{tabular}{|c|c|c|c|c|c|c|c|c|c|c|c|c|c|c|c|c|}
\hline \multicolumn{17}{|c|}{ Ecossistemas } \\
\hline \multirow{2}{*}{ Famílias } & \multicolumn{4}{|c|}{ Fragmento de mata } & \multicolumn{4}{|c|}{ Área agrícola } & \multicolumn{4}{|c|}{ Mata úmida } & \multicolumn{4}{|c|}{ Arajara Park } \\
\hline & F (\%) & D & $\mathbf{A}$ & $\mathbf{C}$ & F (\%) & D & $\mathbf{A}$ & C & F (\%) & D & $\mathbf{A}$ & C & F (\%) & D & $\mathbf{A}$ & C \\
\hline Bibionidae & $1 \%$ & nd & $\mathrm{r}$ & ace & o\% & nd & $\mathrm{r}$ & ace & $\mathrm{o} \%$ & nd & $\mathrm{r}$ & aci & $1 \%$ & nd & $\mathrm{r}$ & ace \\
\hline Bombyliidae & o\% & nd & o & o & o\% & o & o & 0 & o\% & o & o & o & o\% & nd & $\mathrm{r}$ & aci \\
\hline Calliphoridae & $12 \%$ & nd & $\mathrm{d}$ & cte & $10 \%$ & $\mathrm{D}$ & $\mathrm{d}$ & cte & $6 \%$ & nd & $\mathrm{d}$ & cte & $4 \%$ & nd & $\mathrm{c}$ & cte \\
\hline Chloropidae & $3 \%$ & nd & $\mathrm{r}$ & cte & $3 \%$ & $\mathrm{D}$ & ma & cte & $1 \%$ & nd & $\mathrm{c}$ & ace & $3 \%$ & nd & $\mathrm{d}$ & cte \\
\hline Dolychopodidae & о\% & nd & ma & aci & o\% & o & o & o & o\% & o & o & o & o\% & nd & $\mathrm{r}$ & aci \\
\hline Drosophilidae & $23 \%$ & $\mathrm{D}$ & $\mathrm{r}$ & cte & $34 \%$ & $\mathrm{D}$ & $\mathrm{r}$ & cte & $13 \%$ & nd & $\mathrm{d}$ & ace & $37 \%$ & $\mathrm{D}$ & ma & cte \\
\hline Lonchaeidae & $1 \%$ & nd & $\mathrm{r}$ & cte & o\% & nd & abu & cte & o\% & nd & $\mathrm{r}$ & aci & o\% & nd & $\mathrm{r}$ & ace \\
\hline Micropezidae & $0 \%$ & nd & $\mathrm{c}$ & ace & o\% & nd & d & aci & o\% & nd & $\mathrm{ma}$ & ace & $1 \%$ & nd & $\mathrm{r}$ & ace \\
\hline Muscidae & $11 \%$ & nd & $\mathrm{c}$ & cte & $14 \%$ & $\mathrm{D}$ & ma & cte & $4 \%$ & nd & ma & cte & $12 \%$ & $\mathrm{D}$ & $\mathrm{a}$ & cte \\
\hline Otitidae & $14 \%$ & $\mathrm{D}$ & ma & cte & $5 \%$ & nd & d & cte & $29 \%$ & $\mathrm{D}$ & $\mathrm{r}$ & cte & $6 \%$ & nd & $\mathrm{c}$ & cte \\
\hline Sarcophagidae & $30 \%$ & $\mathrm{D}$ & $\mathrm{c}$ & cte & $27 \%$ & $\mathrm{D}$ & $\mathrm{r}$ & cte & $34 \%$ & $\mathrm{D}$ & $\mathrm{d}$ & cte & $29 \%$ & $\mathrm{D}$ & ma & cte \\
\hline Stratiomyiidae & o\% & nd & o & o & o\% & o & o & o & o\% & nd & $\mathrm{c}$ & aci & o\% & nd & $\mathrm{r}$ & aci \\
\hline Tephritidae & $6 \%$ & nd & $\mathrm{ma}$ & cte & $6 \%$ & nd & $\mathrm{r}$ & cte & $3 \%$ & nd & $\mathrm{ma}$ & cte & $6 \%$ & nd & $\mathrm{c}$ & cte \\
\hline Tipulidae & $0 \%$ & nd & $\mathrm{r}$ & aci & $0 \%$ & nd & $\mathrm{r}$ & aci & $10 \%$ & nd & $\mathrm{r}$ & aci & o\% & nd & $\mathrm{r}$ & aci \\
\hline
\end{tabular}

$\mathrm{F}=$ Frequência $\mathrm{D}$ = Dominância: $\mathrm{D}=$ dominante; $\mathrm{nd}=$ não dominante; $\mathrm{A}=$ Abundância: $\mathrm{c}=$ comum; $\mathrm{d}=$ dispersa $;$ ma = muito abundante; $\mathrm{r}=$ rara; $\mathrm{C}=$ Constância: cte $=$ constante $;$ ace $=$ acessória $;$ aci $=$ acidental; $\mathrm{o}=$ sem ocorrência .
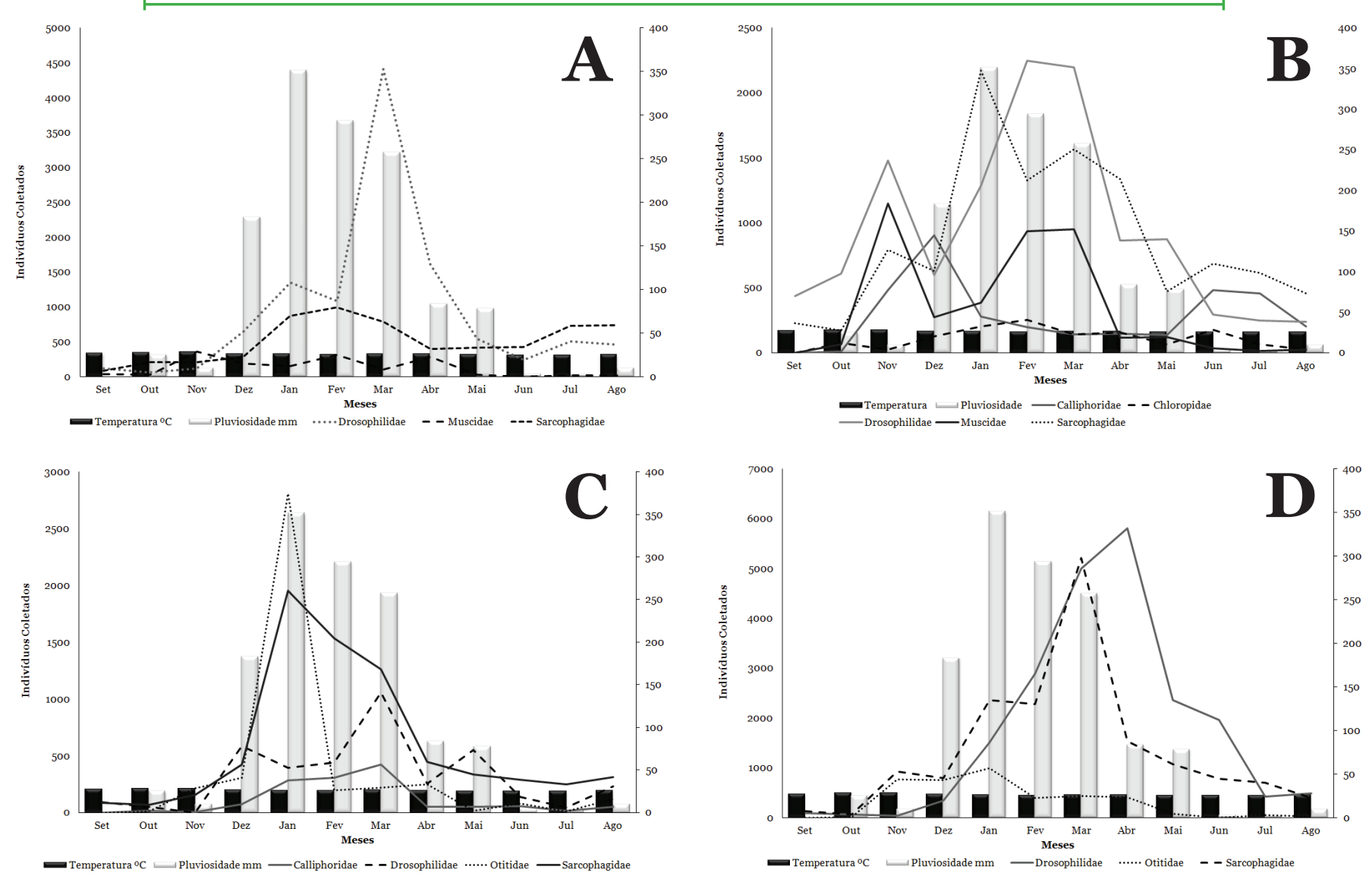

Figura 1. Flutuação populacional das famílias de dípteros que predominaram nos quatro ecossistemas da Área de Proteção Ambiental. A - Arajara Park; B - Área agrícola; C - Mata úmida; D - Fragmento de mata.

Chrysomya tem sido utilizada como bioindicador, devido ao seu alto índice de sinantropia, atuando assim como indicador de ambientes antropizados (BARBOSA et al. 2014).

Os Tephritidae com 6\% de frequência também foram constantes e muito abundantes; são insetos conhecidos como moscas das frutas, pois são frugívoras, atacando um grande número de frutos hospedeiros, cultivados e silvestres. No entorno do fragmento, os produtores familiares cultivam frutas tropicais, dentre as quais se destaca a goiaba. De acordo com AzEvEDo et al. (2010), as espécies Anastrepha zenildae Zucch, Anastrepha obliqua (Macquart),
Anastrepha fraterculus (Wiedemann), Anastrepha sororcula Zucch e Ceratitis capitata (Wiedemann) são comuns no Cariri, atacando não somente essa fruta, mas também outras da região. As demais famílias apresentaram baixíssimas frequências e foram não dominantes, dispersas e de ocorrência acidental e algumas estiveram ausentes (Tabela 2).

$\mathrm{Na}$ área agrícola, os Drosophilidae foram mais frequentes (34\%) (Tabela 2), pelo fato desse ecossistema apresentar um maior número de frutos oriundos de fruteiras cultivadas em processo de fermentação, quando comparado com o fragmento, onde 
só existiam praticamente frutos silvestres. A maior densidade populacional ocorreu no mês de fevereiro devido a uma maior pluviosidade $(294,2 \mathrm{~mm})$ associada a uma menor temperatura $\left(25,4{ }^{\circ} \mathrm{C}\right)$. Alguns drosofilídeos são endêmicos de certas regiões e outros são cosmopolitas, sendo que muitas vezes se dispersam em associação às atividades humanas (Tidon-Sklorz \& SENE 1999). Isso explica a maior porcentagem desses insetos nessa área, onde há a intervenção humana no cultivo de fruteiras.

Os Sarcophagidae (27\%) apresentaram frequência próxima à do fragmento (30\%), já que nessa área existiam muitos animais domésticos como porcos, gado, jegues, cavalos, galinhas, patos e outros. Os Muscidae e Calliphoridae também demonstraram frequências próximas às do fragmento (Tabela 2), em virtude também da presença desses animais, já que são insetos de importância médico-veterinária (GALLO et al. 2002).

Essas quatro famílias foram dominantes e constantes, mas somente Muscidae foi muito abundante devido à proximidade da área com as residências e animais domésticos. As moscas das frutas apresentaram a mesma frequência do fragmento e os Chloropidae, apesar da baixa frequência (3\%), dominaram a área, foram muito abundantes e constantes (Tabela 2). São conhecidas como moscas "lambe olhos" e se alimentam de secreções de animais (Triplehorn \& JoHNSON 2011), o que explica os altos índices faunísticos dessa família.

Os Otitidae (5\%), apesar de constantes, devido à presença de capim elefante e outras gramíneas para alimentar o gado, foram não dominantes e dispersos na área, enquanto que as demais famílias estiveram ausentes (Tabela 2).

Na mata úmida, a família mais frequente foi Sarcophagidae (34\%) (Tabela 2), devido à presença de carcaças de animais silvestres mortos em processo de decomposição. Também com maiores picos populacionais nos meses de janeiro e fevereiro (Figura 1). A porcentagem de drosofilídeos foi menor em virtude da menor quantidade de frutos em fermentação, quando comparado com o fragmento e a área agrícola. Os Tipulidae apresentaram 10\% de frequência e vivem principalmente em habitats úmidos e com vegetação abundante (TRIPLEHORN \& JOHNSON 2011), condições estas encontradas na mata úmida. Ao coletar dípteros em um fragmento de transição entre cerrado e mata úmida, TAUHYL \& GuIMARÃEs (2012), constataram que a família Tipulidae estava entre as mais frequentes ao longo das quatro estações do ano, junto com outras famílias, exemplificando a complexidade e distribuição das famílias ao longo do ano, corroborando com os resultados obtidos por Silva et al. (2011). As moscas-das-frutas apesar de muito abundantes e constantes apresentaram a metade da frequência dos ecossistemas anteriores e não dominaram nesse ecossistema, devido à menor quantidade de frutos silvestres. As demais famílias apresentaram baixíssimas frequências e foram não dominantes, dispersas e de ocorrência acidental e algumas não estiveram presentes (Tabela 2).

No Arajara Park, a família mais frequente foi Drosophilidae (37\%), com frequência próxima à da área agrícola (34\%), haja vista que nesse ecossistema a presença de turistas trazendo ou consumindo frutas atrai esses insetos, indicando ação antrópica, seguida dos Sarcophagidae (29\%), com frequências similares à dos ecossistemas anteriores, assim como os Muscidae (12\%) e Tephritidae (6\%) (Tabela 2). As três primeiras famílias foram dominantes, mas somente Drosophilidae e Sarcophagidae foram muito abundantes e todas foram constantes. As demais famílias apresentaram baixíssimas frequências e foram não dominantes, dispersas e de ocorrência acidental e algumas estiveram ausentes (Tabela 2).

Índices faunísticos dos dípteros coletados na estação chuvosa. Nessa estação e nos quatro ecossistemas foi amostrado um total de 11 famílias da ordem Diptera, três a menos do que foi encontrado na estação seca, sendo a família Drosophilidae a mais numerosa, seguida de Sarcophagidae (Tabela 3). Essa diminuição da abundância amostrada pode ser explicada pela presença de acontecimentos atípicos, conforme citado por OLIVEIRA et al. (2008), visto que a literatura científica registra que em várias comunidades de insetos, as suas populações e riquezas, tendem a aumentar com o início das chuvas, bem como o início de suas atividades, de acordo com Wolda (1988). Esse aumento da atividade pode ser exemplificado pelo aumento da abundância da família Drosophilidae, visto que se capturou quase o dobro de drosofilídeos (30.550) quando comparado com a estação seca (16.495), o que indica que esses insetos preferem ambientes úmidos. Já os sarcofagídeos, califorídeos e tefritídeos apresentaram números semelhantes entre as estações, não apresentando, portanto, preferência por uma delas. Dos 70.352 indivíduos capturados na estação chuvosa, 46,63\% foram encontrados no fragmento, 22,31\% no Arajara Park, 20,32\% na área agrícola e 10,74\% na mata úmida. Observa-se nessa estação um menor número de famílias, mas, no entanto, foi encontrado um maior número de indivíduos da ordem Diptera.

No fragmento, os dípteros da família Drosophilidae foram os mais frequentes (49\%), seguido por Sarcophagidae (29\%). Os maiores picos populacionais dessas famílias deu-se nos meses de março e abril em condições climáticas de altas precipitações (257,6 e $84 \mathrm{~mm}$, respectivamente) e temperaturas elevadas (26,2 e $26^{\circ} \mathrm{C}$, respectivamente). Nessa estação e nesse ecossistema, os drosofililídeos dobraram a sua frequência, quando comparada com a estação seca (23\%), enquanto que os sarcofagídeos permaneceram praticamente com a mesma frequência (30\%). Essas duas famílias foram dominantes e constantes, enquanto que a primeira foi rara e a segunda dispersa (Tabela 4).

Os Calliphoridae só apresentaram 7\% de frequência, quase a metade da encontrada na estação seca que foi de $12 \%$, ocorrendo a metade para os Tephritidae. A literatura registra que os califorídeos sofrem forte influência sazonal das variações climáticas (FERREIRA \& LACERDA 1993). Ao delimitar os efeitos da sazonalidade para populações de Cochliomyia macellaria (Fabricius) (Calliphoridae) em um ecossistema de Cerrado, Gomes et al. (2000), encontrou picos populacionais durante o aumento da temperatura e início das chuvas. Situação semelhante da encontrada na presente pesquisa. Contudo, por sua vez, para um ecossistema inserido em um fragmento florestal no Rio de Janeiro não foi encontrada correlação positiva entre a abundância dos califorídeos e a pluviosidade (FERRAz et al. 2010). Acredita-se que essa família tenha preferência pela estação seca ao invés da chuvosa, contrariando com os resultados obtidos por LEANDRO \& D'ALMEIDA (2005), no qual a maior abundância foi obtida no período chuvoso de sua respectiva área de pesquisa. As demais famílias apresentaram baixíssimas frequências e foram não dominantes, dispersas e de ocorrência acidental e algumas não estiveram presentes.

A ausência de correlação positiva entre a variação da abundância em função da temperatura e pluviosidade contrasta com os resultados obtidos por TAUHYL \& GUIMARÃES (2012), no qual os autores encontraram um aumento significativo da abundância de dípteros em função da temperatura e pluviosidade, indicando como uma possível justificativa no aumento das condições de forrageamento na área de pesquisa. Contudo, tratando-se de grupos específicos como a família Calliphoridae, pode-se observar que o aumento da precipitação diminui a densidade e a atividade (MARINHo et al. 2006).

No Arajara Park, novamente os dípteros da família Drosophilidae (50\%) e Sarcophagidae (22\%) foram os mais frequentes, no entanto, os Tephritidae dobraram a sua frequência, atingindo $7 \%$. Isso se deve provavelmente à presença de frutos silvestres e/ ou cultivados em processo de maturação já que é um ecossistema que sofreu ação do homem. As demais famílias apresentaram baixíssimas frequências e foram não dominantes, dispersas e de ocorrência acidental e algumas estiveram ausentes (Tabela 4). 
Tabela 3. Número total de indivíduos das famílias de Diptera capturadas na estação chuvosa nos quatro ecossistemas da Área de Proteção Ambiental do Araripe. Barbalha-CE, de 01 de março a 30 de agosto de 2011.

\begin{tabular}{|c|c|c|c|c|c|}
\hline \multirow[b]{2}{*}{ Famílias } & \multirow[b]{2}{*}{$\begin{array}{c}\text { Fragmento de } \\
\text { mata }\end{array}$} & \multicolumn{4}{|c|}{ Ecossistemas } \\
\hline & & Arajara Park & Área agrícola & Mata úmida & Total \\
\hline Bibionidae & 278 & 278 & 156 & 8 & 720 \\
\hline Calliphoridae & 2.400 & 694 & 1.358 & 643 & 5.095 \\
\hline Chloropidae & 655 & 501 & 603 & 482 & 2.241 \\
\hline Drosophilidae & 1.6031 & $7 \cdot 770$ & 4.473 & 2.276 & 30.550 \\
\hline Lonchaeidae & 68 & 15 & 73 & 2 & 158 \\
\hline Micropezidae & 742 & 591 & 306 & 116 & 1.755 \\
\hline Muscidae & 574 & 440 & 1.230 & 206 & 2.450 \\
\hline Otitidae & 981 & 446 & 574 & 683 & 2.684 \\
\hline Sarcophagidae & 9.675 & 3.491 & 4.666 & 2.887 & 20.719 \\
\hline Tephritidae & 1.075 & 1.125 & 800 & 202 & 3.202 \\
\hline Tipulidae & 325 & 343 & 57 & 53 & 778 \\
\hline Total & 32.804 & 15.694 & 14.296 & 7.558 & 70.352 \\
\hline
\end{tabular}

Tabela 4. Análise faunística das famílias de Diptera capturados na estação chuvosa em quatro ecossistemas da Área de Proteção Ambiental do Araripe, Barbalha-CE, de 01 de março a 30 de agosto de 2011.

\begin{tabular}{|c|c|c|c|c|c|c|c|c|c|c|c|c|c|c|c|c|}
\hline \multirow{3}{*}{ Famílias } & \multicolumn{16}{|c|}{ Ecossistemas } \\
\hline & \multicolumn{4}{|c|}{ Fragmento de mata } & \multicolumn{4}{|c|}{ Arajara Park } & \multicolumn{4}{|c|}{ Área agrícola } & \multicolumn{4}{|c|}{ Mata úmida } \\
\hline & F (\%) & $\mathbf{D}$ & $\mathbf{A}$ & c & F (\%) & $\mathbf{D}$ & $\mathbf{A}$ & $\mathbf{C}$ & F (\%) & $\mathbf{D}$ & $\mathbf{A}$ & $\mathbf{C}$ & F (\%) & $\mathbf{D}$ & $\mathbf{A}$ & C \\
\hline Bibionidae & $1 \%$ & nd & $\mathrm{r}$ & cte & $2 \%$ & nd & d & cte & $1 \%$ & nd & $\mathrm{r}$ & cte & o\% & nd & $\mathrm{r}$ & ace \\
\hline Calliphoridae & $7 \%$ & nd & $\mathrm{r}$ & cte & $4 \%$ & nd & $\mathrm{d}$ & cte & $9 \%$ & $\mathrm{D}$ & d & cte & $9 \%$ & $\mathrm{D}$ & $\mathrm{d}$ & cte \\
\hline Chloropidae & $2 \%$ & nd & $\mathrm{r}$ & cte & $3 \%$ & nd & d & cte & $4 \%$ & nd & $\mathrm{ma}$ & cte & $6 \%$ & nd & ma & cte \\
\hline Drosophilidae & $49 \%$ & $\mathrm{D}$ & $\mathrm{r}$ & cte & $50 \%$ & $\mathrm{D}$ & ma & cte & $31 \%$ & $\mathrm{D}$ & d & cte & $30 \%$ & $\mathrm{D}$ & d & cte \\
\hline Lonchaeidae & o\% & nd & $\mathrm{r}$ & cte & o\% & nd & $\mathrm{r}$ & ace & $1 \%$ & nd & $\mathrm{c}$ & cte & o\% & nd & $\mathrm{d}$ & cte \\
\hline Micropezidae & $2 \%$ & nd & $\mathrm{r}$ & cte & $4 \%$ & nd & d & cte & $2 \%$ & nd & d & cte & $2 \%$ & nd & $\mathrm{d}$ & cte \\
\hline Muscidae & $2 \%$ & nd & $\mathrm{r}$ & aci & $3 \%$ & nd & $\mathrm{d}$ & cte & $9 \%$ & $\mathrm{D}$ & ma & cte & $3 \%$ & nd & $\mathrm{ma}$ & cte \\
\hline Otitidae & $3 \%$ & nd & ma & cte & $3 \%$ & nd & d & cte & $4 \%$ & nd & d & cte & $9 \%$ & $\mathrm{D}$ & $\mathrm{r}$ & cte \\
\hline Sarcophagidae & $29 \%$ & $\mathrm{D}$ & $\mathrm{d}$ & cte & $22 \%$ & $\mathrm{D}$ & $\mathrm{ma}$ & cte & $33 \%$ & $\mathrm{D}$ & $\mathrm{r}$ & cte & $38 \%$ & $\mathrm{D}$ & $\mathrm{d}$ & cte \\
\hline Tephritidae & $3 \%$ & nd & $\mathrm{ma}$ & cte & $7 \%$ & nd & $\mathrm{c}$ & cte & $6 \%$ & nd & $\mathrm{r}$ & cte & $3 \%$ & nd & ma & cte \\
\hline Tipulidae & $1 \%$ & nd & $\mathrm{r}$ & aci & $2 \%$ & nd & $\mathrm{d}$ & aci & o\% & nd & $\mathrm{r}$ & aci & $1 \%$ & nd & $\mathrm{r}$ & aci \\
\hline
\end{tabular}

$\mathrm{F}$ = Frequência; $\mathrm{D}=$ Dominância: $\mathrm{D}$ = dominante; $\mathrm{nd}=$ não dominante; $\mathrm{A}=$ Abundância: $\mathrm{c}=$ comum; $\mathrm{d}$ = dispersa; ma = muito abundante; $\mathrm{r}=$ rara; $\mathrm{C}=$ Constância: cte $=$ constante $;$ ace $=$ acessória $;$ aci $=$ acidental $; \mathrm{o}=$ sem ocorrência .

Na área agrícola novamente os dípteros da família Drosophilidae (31\%) e Sarcophagidae (33\%) foram os mais frequentes, com frequências bem próximas uma da outra, mas com redução da primeira e aumento da segunda família, quando comparado com o Arajara Park, também com maiores picos populacionais nos meses de março e abril (Figura 1). Os Calliphoridae e Muscidae apresentaram a mesma frequência (9\%) e os Tephritidae praticamente mantiveram a sua (6\%) (Tabela 4). Tal redução da frequência observada para Calliphoridae e Muscidade pode ser justificada pela abundância de Sarcophagidae, visto que tal família promove reduções significativas em dípteros encontrados em fezes de gado, como demonstrado por Mendes \& LinHares (2002).

As quatro primeiras famílias foram dominantes e constantes, mas somente Muscidae foi muito abundante em virtude da proximidade da área com residências e criações de animais, indicando ambiente modificado pelo homem, concordando com o trabalho de LEANDRo \& D'Almeida (2005). Apesar da baixa frequência dos Chloropidae (4\%), também foram muito abundantes e constantes, já que se alimentam de secreções de animais que são criados na área agrícola, indicando a presença de animais no ecossistema avaliado em condição chuvosa.

Na mata úmida os dípteros da família Sarcophagidae foram mais frequentes (38\%), devido à presença de carcaças de animais silvestres mortos, com maior pico populacional no mês de março (Figura 1), seguida de Drosophilidae (30\%) devido aos frutos silvestres em processo de fermentação, com frequências próximas às da área agrícola (Tabela 4). Os Calliphoridae e Otitidae apresentaram a mesma frequência ( $9 \%$ ) e os Chloropidae atingiram a maior frequência nesses ecossistemas (6\%), quando comparado com os demais.

Os Sarcophagidae e Drosophilidae são as famílias mais abundantes tanto na estação seca como na chuvosa, nos quatro ecossistemas da Área de Proteção Ambiental do Araripe.

Os Sarcophagidae dominam e ocorrem frequente e constantemente em todos os ecossistemas da estação seca da Área de Proteção Ambiental do Araripe, enquanto que os Drosophilidae apesar de dominarem e ocorrerem constantemente, são mais frequentes na área agrícola e no Arajara Park devido à ação antrópica que altera esses ecossistemas para a implantação de culturas agrícolas e áreas de lazer.

Os Drosophilidae dominam e ocorrem frequente e constantemente em todos os ecossistemas da estação chuvosa da Área de Proteção Ambiental do Araripe, enquanto que os Sarcophagidae, apesar de dominarem e ocorrerem constantemente, são mais frequentes na área agrícola e na mata úmida devido à ação antrópica obtida pelas irrigações das culturas agrícolas da área agrícola associada com maior umidade pelo microclima favorável encontrado na mata úmida. 
Os picos populacionais dos dípteros que predominam nos ecossistemas da Área de Proteção Ambiental do Araripe ocorrem nos meses chuvosos associados a baixas temperaturas.

\section{AGRADECIMENTOS}

Ao Chefe da Área de Proteção Ambiental do Araripe, William Brito, pela disponibilização das áreas para a realização das coletas. Ao analista ambiental Carlos Neto, pelo acompanhamento da pesquisa nessas áreas.

\section{REFERÊNCIAS}

Anjos, A.F. \& M.A. Navarro-Silva, 2008. Culicidae (Insecta: Diptera) em área de Floresta Atlântica, no Estado do Paraná, Brasil. Acta Scientarum Biological Sciences, 30: 23-27.

Azevedo, F.R., J.A Guimarães, A.A.F. Simplício \& H.R. Santos, 2010. Análise faunística e flutuação populacional de moscasdas-frutas (Diptera: Tephritidae) em pomares comerciais de goiaba na região do Cariri cearense. Arquivos do Instituto Biológico, 77: 33-41.

Azevedo, F.R., M.A.R. Moura, M.S.B., Arrais \& D.R Nere, 2011. Composição da Entomofauna da Floresta Nacional do Araripe em diferentes vegetações e estações do ano. Revista Ceres Viçosa, 58: 740-748.

Barbosa, L.S., A.M. Cunha, M.S. Couri \& V.C. Maia, 2014. Muscidae, Sarcophagidae, Calliphoridae e Mesembrinellidae (Diptera) da Estação Biológica de Santa Lúcia (Santa Teresa, Espírito Santo, Brasil). Boletim do Museu Biológico Mello Leitão, 33: 131-140.

Bittencourt, A.J. \& G.E. Moya Borja, 200o. Stomoxys calcitrans (L.): Preferência por regiões do corpo de eqüinos para alimentação. Parasitología Día, 24: 119-122.

Braack, L.E.O., 1987. Community dynamics of carrion-attendant arthopods in tropical African woodland. Oecologia, 72: 402409.

Buzzi, Z.J., 2008. Entomologia didática. 4 ed. Curitiba, Editora UFPR, $348 \mathrm{p}$.

Catts, E.P. \& M.L. Goff, 1992. Forensic entomology in criminal investigations. Annual Review of Entomology, 37: 253-272.

Dias, E.S., D.P. Neves \& H.S. Lopes, 1984. Estudos sobre a fauna de Sarcophagidae (Diptera) de Belo Horizonte, Mingas Gerais - Variação Sazonal. Memórias do Instituto Oswaldo Cruz, 79: 409-412.

Ferraz, A.C.P., B.Q. Gadelha \& V.M. Aguiar-Coelho, 2010. Influência climática e antrópica na abundância e riqueza de Calliphoridae (Diptera) em um fragmento florestal da Reserva Biológica do Tinguá, RJ. Neotropical Entomology, 39: 476-485.

Ferraz, A.C.P., 2011. Efeitos de borda em florestas tropicais sobre artrópodes, com ênfase nos dípteros ciclorrafos. Oecologia Australis, 15: 189-198.

Ferreira, M.J.M. \& P.V. Lacerda, 1993. Muscóides sinantrópicos associados ao lixo urbano em Goiânia, Goiás. Revista Brasileira de Zoologia, 10: 185-195.

Furusawa, G.P. \& P.C.R. Cassino, 2006. Ocorrência e Distribuição de Calliphoridae (Diptera: Oestroidea) em um Fragmento de Mata Atlântica Secundária no Município de Engenheiro Paulo de Frontin, Médio Paraíba, RJ. Revista de Biologia e Ciências da Terra, 6: 152-164.

Gallo, D., O. Nakano, S. Silveira Neto, R.P.L. Carvalho, G.C. Batista, E. Berti Filho, J.R.P. Parra, R.A. Zucchi, S.B. Alves, J.D. Vendramim, L.C. Marchini \& J.R.S. Lopes, 2002. Entomologia agrícola. Piracicaba, FEALQ, $920 \mathrm{p}$

Gomes, A., W.W. Koller \& A.T.M. Barros, 2000. Sazonalidade da mosca-varejeira, Cochliomyia macellaria (Diptera: Calliphoridae), na região dos Cerrados, Campo Grande, MS. Revista Brasileira de Parasitologia Veterinária, 9: 125-128.

Greenberg, B., 1973. Flies and disease: biology and disease transmission. New Jersey, Princeton University, 447 p.

Guimarães J. H., N.A. Papavero \& A.P. Prado. 1983. As miíases na região Neotropical (identificação, biologia e bibliografia).
Revista Brasileira de Zoologia, 1: 293-416.

Leandro, M.J.F. \& J.M. D’Almeida, 2005. Levantamento de Calliphoridae, Fanniidae, Muscidae e Sarcophagidae em um fragmento de mata na Ilha do Governador, Rio de Janeiro, Brasil. Ilheringia, Série Zoológica, 35: 377-381.

Marinho, C.R., L.S. Barbosa, A.C.G. Azevedo, M.M.C. Queiroz, M.A. Valgode \& V.M. Aguiar-Coelho, 2006. Diversity of Calliphoridae (Diptera) in Brazil’s Tinguá Biological Reserve. Brazilian Journal of Biology, 66: 95-100.

Mendes, J. \& A.X. Linhares, 2002. Cattle Dung Breeding Diptera in Pastures in Southeastern Brazil: Diversity, Abundance and Seasonality. Memórias do Instituto Oswaldo Cruz, 97: 37-42.

Moura, M. O., C.J.B. Carvalho \& E.L.A. Monteiro-Filho. 1997. A preliminary analysis of insects of medico-legal importance in Curitiba, States of Paraná. Memórias do Instituto Oswaldo Cruz, 92: 269-274.

Neves, D.P., 2005. Parasitologia Humana. São Paulo, Editora Atheneu, $494 \mathrm{p}$.

Oliveira, R.C., A.R. Fonseca. \& C.G. Silva, 2008. Fauna de dípteros em uma área de Cerrado no interior de Divinópolis, estado de Minas Gerais. Revista Trópica: Ciências Agrárias e Biológicas, 2: 3-7.

Pereira, V.L., 2008. Padrões populacionais de moscas frugívoras (Diptera: Tephritoidea) em um fragmento de floresta semidecídua e em um pomar comercial na Região de Dourados - MS, Brasil. Dissertação (Mestrado em Entomologia e Conservação da Biodiversidade) Universidade da Grande Dourados. 46p.

Santos, C.A.M., F.R. Azevedo, E.S. Moura, D.R. Nere \& R. Azevedo, 2012. Uso da armadilha Mcphail na captura de insetos em vegetações da Floresta Nacional do Araripe. Encontro Universitário da UFC no Cariri, 4. Resumo expandido. 1-5, Universidade Federal do Ceará, Juazeiro do Norte-CE.

Silva, M. S., J.C. Fontenelle \& R.P. Martins, 2001. Por que moscas visitam flores? Ciência Hoje, 30: 68-71.

Silva, N.A.P., M.R. Frizzas \& C.M. Oliveira, 2011. Seasonality in insect abundance in the "Cerrado" of Goiás State, Brazil. Revista Brasileira de Entomologia, 55: 79-87.

Silveira Neto, S., O. Nakano, D. Bardin \& N.A. Vila Nova, 1976. Manual de ecologia dos insetos. São Paulo, Agronômica Ceres, $420 \mathrm{p}$.

Silveira Neto, S., R.C. Monteiro, R.A. Zucchi \& R.C.B. Moraes, 1995. Uso da análise faunística de insetos na avaliação do impacto ambiental. Scentia Agrícola, 52:9-15.

Smith, K.G.V., 1986. A manual of forensic entomology. Ithaca, Cornell University Press, 205 p.

Souto, K. C.F.L., C.D. Pereira \& C. Lomônaco, 2011. Body Size, Symmetry and Abundance of Euxesta stigmatias (Loew) and Euxesta sororcula (Wiedemann) (Diptera: Ulidiidae) in a Natural Reserve and in a Guava Orchard in Uberlândia, MG, Brazil. Neotropical Entomology, 40: 661-668.

Steyskal, G.C., 1977. History and use of the McPhaill trap. Florida Entomologist, 60: 11-16.

Tauhyl, L.G.M. \& M.V.U. Guimarães, 2012. Dipterofauna de fragmentos vegetacionais da UFSCar - Campus Sorocaba, SP, Brasil. Revista Trópica: Ciências Agrárias e Biológicas, 6: 79-88.

Tidon-Sklorz, R. \& F.M. Sene, 1999. Drosophila, p. 246-261. In: Brandão, C. R. F. \& E. M. Cancello (Eds.). Biodiversidade do Estado de São Paulo, Brasil: síntese do conhecimento ao final do século XX: invertebrados terrestres. São Paulo, FAPESP, $279 \mathrm{p}$.

Triplehorn, C.A. \& N.F. Johnson, 2011. Estudo dos insetos. São Paulo, Cengage Learning, $809 \mathrm{p}$.

Uchôa-Fernandes M.A., I. Oliveira, R.M.S. Molina \& R.A. Zucchi, 2003. Populational fluctuation of frugivorous flies (Diptera: Tephritoidea) in two orange groves in the state of Mato Grosso do Sul, Brazil. Neotropical Entomology, 32: 19-25.

Wink, C., J.V.C Guedes, C.K., Fagundes, A.P., Rovedder, 2005. Insetos edáficos como indicadores da qualidade ambiental. Revista de Ciências Agroveterinárias, 4: 60-71. 
Wolda, H., 1988. Insect seazonality: why? Annual Review of Ecology and Systematics, 19: 1-18.
Recebido em:17/o2/2014

Aceito em: 05/o3/2015

Como citar este artigo:

Azevedo, F.R., R.A. Azevedo, C.A.M. Santos, E.S. Moura \& D.R. Nere, 2015. Análise Faunística e Flutuação Populacional da Dipterofauna de Ecossistemas da Área de Proteção Ambiental do Araripe, Barbalha, CE. EntomoBrasilis, 8 (2): 117-124.

Acessível em: doi:10.12741/ebrasilis.v8i2.423
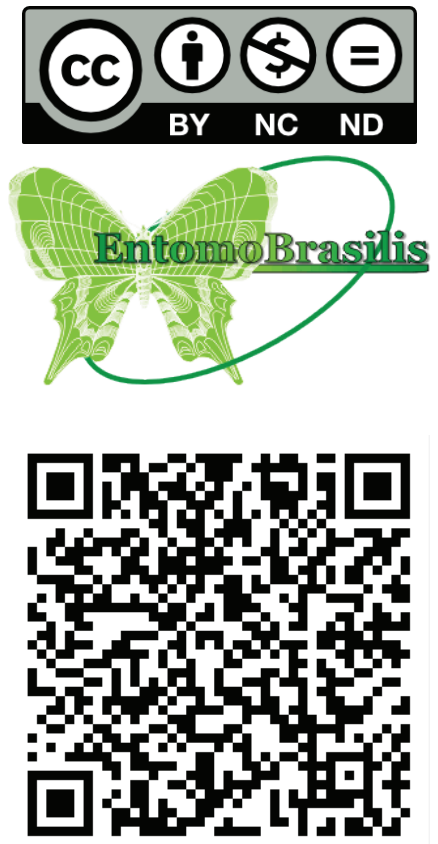